\title{
Health care needs of Jordanian caregivers of patients with cancer receiving chemotherapy on an outpatient basis
}

M.S. Al-Jauissy'

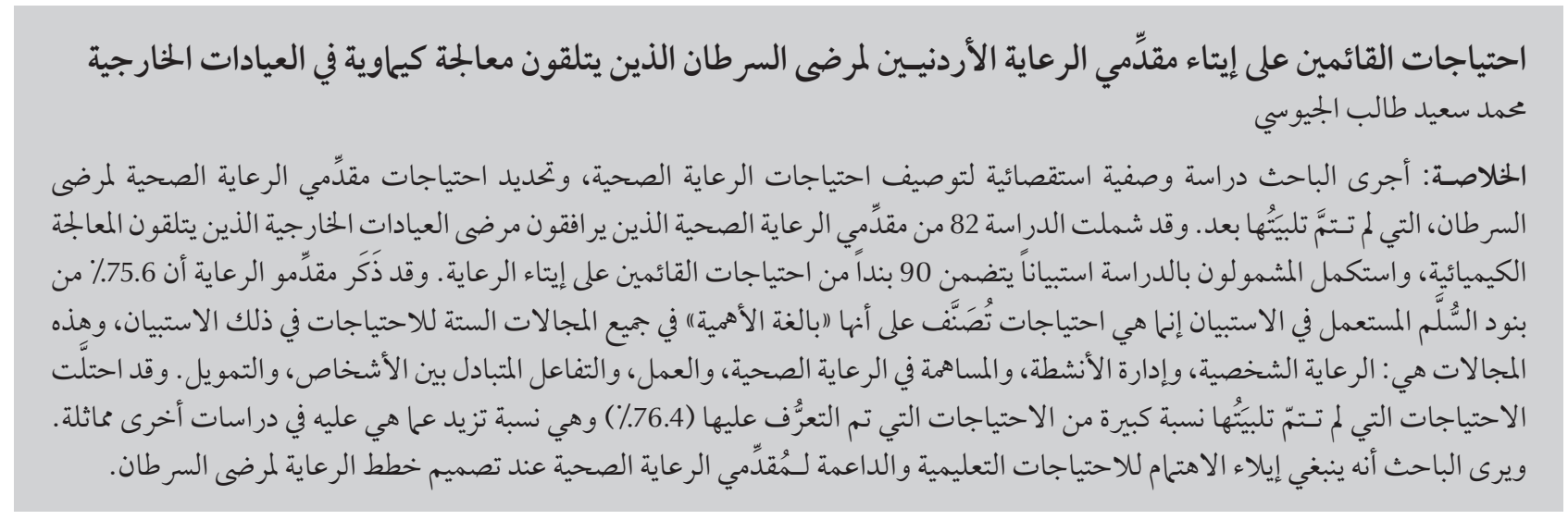

ABSTRACT This descriptive exploratory study was conducted to describe the health care needs and identify unmet needs of the caregivers of cancer patients in Jordan. A total of 82 caregivers accompanying patients to an outpatient chemotherapy clinic completed the 90-item caregiver need scale. Caregivers reported $75.6 \%$ of scale items as needs and rated these as "very important" needs on all 6 areas of the caregivers' need scale: personal care, activity management, involvement with health care, work, interpersonal interaction and finance. Unmet needs of caregivers were a higher proportion of identified needs $(76.4 \%)$ than in similar studies elsewhere. The education and support needs of caregivers need to be considered when designing care plans for cancer patients.

Besoins en soins de santé des soignants jordaniens de patients cancéreux recevant une chimiothérapie en consultation externe

RÉSUMÉ Cette étude exploratoire et descriptive a été menée pour décrire les besoins en soins de santé des aidants de patients cancéreux en Jordanie et identifier leurs besoins non satisfaits. Au total, 82 aidants accompagnant des patients dans un service de chimiothérapie ambulatoire ont rempli le questionnaire sur l'échelle des besoins des aidants en 90 items. Ils ont désigné 75,6 \% des items de l'échelle comme étant des « besoins », qu'ils ont qualifiés de «très importants », dans l'ensemble des six domaines de l'échelle des besoins des aidants : soins personnels, gestion de l'activité, implication dans les soins de santé, travail, communication interpersonnelle et finances. Les besoins non satisfaits des aidants représentaient un pourcentage des besoins identifiés (76,4 \%) supérieur à celui d'autres études similaires. Les besoins en formation et en soutien des aidants doivent être pris en compte lors de l'élaboration des plans de soins pour les patients cancéreux. 


\section{Introduction}

There is a growing interest in shortening hospital stays for patients with cancer and treating them on an outpatient basis [1-3]. This shift of care from hospital to the home places considerable responsibility on informal family caregivers. In fact, researchers reported that caregivers assume approximately $50 \%-55 \%$ of the total care required for patients with cancer at home $[4,5]$.

Caring for patients with cancer is a complex and demanding role $[1,6,7]$. Cancer caregivers are expected to meet patients' needs in the physical, psychological, social, spiritual and financial dimensions [6,7]. In addition, caregivers need to balance the responsibilities of caregiving role with their own needs and demands, which is one of the most challenging problems facing caregivers of patients with cancer [1].

Previous research demonstrated that caregivers have to commence this role suddenly with limited knowledge and experiences, with a negative effect on the patient and the caregiver $[1,6]$. For example, in a prospective population-based cohort study, it was found that stress associated with caring for a patient with cancer increased the caregiver's risk of mortality by $63 \%$ within 5 years [8]. In addition, the role of caregiving for patients with cancer was found to be associated with sleep disturbance $[9,2]$, fatigue [10-12], anxiety [13], social withdrawal $[4,14]$, depression $[13,15]$, impaired quality of life [2] and financial constraints $[2,15]$. Designing individualized care plans which incorporate caregivers' needs is an essential element to improve the quality of care provided to patients with cancer $[4,16]$.

In Jordan, the number of people providing care for patients with cancer at home is unknown and the needs of caregivers have never been examined before from a Jordanian Arab perspective. This study was therefore designed to address the following research questions: what are the health care needs identified by Jordanian caregivers of cancer patients receiving chemotherapy on an outpatient basis; how do these caregivers rank the importance of those identified needs; and are the identified needs currently being met?

\section{Methods}

\section{Sample}

Consecutive primary caregivers accompanying cancer patients to an outpatient chemotherapy clinic at a major university-affiliated hospital in Jordan during the period 10 August to 25 October 2006 were selected to participate in this study. Since this was an exploratory, descriptive study, no attempt was made to estimate sample size. The inclusion criteria for caregivers were: Jordanian nationality; male or female; identified by the patient as the individual who is most involved in, or affected by, the patient's situation; residing in the patient's home; able to communicate in Arabic; and able to give written consent.

\section{Instruments}

Data were collected using the following scales: the caregivers' demographic data sheet, the caregivers' medical information form and the caregivers' need scale.

The demographic data sheet contained questions related to participants' characteristics such as: age, sex, marital status, income level, number of people living in the same household and education. Two items were added to reflect structural barriers to health services: distance travelled to the hospital and usual time required to travel from home to hospital.

The medical information form contained questions related to the caregivers' chronic medical conditions, physical disabilities andhistory ofmental illnesses. In addition, participants were asked to report the presence of negative impacts thought to be associated with the caregiving role, including sleep disturbances, fatigue, anxiety, distress, financial constraints and depression.

The caregivers' need scale was developed to assess caregivers' current health care needs in relation to the patient's situation and contains 6 subscales and 90 items [17]. These subscales are: personal care (28 items), activity management (13 items), involvement with health care (19 items), work (2 items), inter-personal interaction (21 items) and finance ( 7 items). Each item is measured on a $10 \mathrm{~cm}$ visual scale from "not a need at all" (0) to "very big need" (10). In addition, participants are asked to indicate whether each need is being met or not. The caregivers' need scale yields 3 scores for each item: the existence of the need (an item is considered as an existing need if $66 \%$ or more of the participants report it as aneed); the need was met or not met (participants mark a "need met" or "need unmet" column for each need they decide to be existing); and the importance of the need (an item is considered "very important" if $50 \%$ or more of the participants rank it as 9 or 10 on the need scale).

The original author reported that the caregivers' need scale had 99\% inter-rater reliability and a reliability coefficient alpha of 0.80 . Construct and face validity for the scale were also established [17].

\section{Procedure}

A panel of 2 Masters level nurses and 2 lay persons who were competent in English and Arabic languages translated and back-translated the caregivers' need scale. Any discrepancy between the original version and the translated form was resolved based on the suggestions of the researchers and the panel.

Permission from the institutional review board and the hospital administration was obtained before starting data collection. Then the researchers visited the clinic on a daily basis during the study period to recruit participants. Once a participant was identified the researcher approached the patient and 
caregivers to discuss the purpose and procedure of the study, invite them to participate and obtain written consent. Participants were assured that confidentiality would be maintained throughout the study and that the results would be reported as aggregates without revealing their identity. In addition, participants were assured that they could withdraw from the study at any time without jeopardizing their care at the institution.

Astructured interview lasting 30-35 minutes was used to collect data from each participant. Completed forms were coded and fed into a computer for data analysis. A pilot test of the translated version of the caregivers' need scale was made with 10 participants before the beginning of the study, resulting in an alpha coefficient of 0.86 .

\section{Results}

\section{Participants' demographic characteristics}

Of the 104 companions of patient who were approached and invited to participate, 22 (21.2\%) did not meet the inclusion criteria and were excluded. Table 1 summarizes the demographic characteristics of the remaining 82 participants. The majority of participants were female $(64,78.1 \%)$ and married (58, 70.7\%). The education level of participants ranged from 2 to 18 years, with a mean of 8.4 [standard deviation (SD) 12.6] years. The age of participants ranged from 21 to 62 years, with a mean of 36.2 (SD 15.8) years. The distance between participants' home and hospital ranged from 20 to 60 miles, mean 32.5 (SD 14.6) miles, and the mean time required to travel from home to hospital was 64.0 (SD 17.4) minutes.

\section{Participants' medical status}

Participants reported suffering from 5 chronic medical conditions: diabetes mellitus (34.2\%), hypertension (36.6\%), angina (29.3\%), renal failure (3.7\%) and rheumatoid arthritis (2.4\%).
Negative impacts of the caregiving role experienced by participants were: fatigue $(78.0 \%)$, sleep disturbance (64.6\%), anxiety (60.7\%), financial constraints (58.5\%) and mood disturbance (43.9\%). None of the participants reported having major physical disabilities or a history of psychiatric illness.

\section{Participants' existing needs}

Two-thirds of the participants ( $n=55$, $67.1 \%)$ identified a total of 68 needs, i.e. $75.6 \%$ of the 90 items found on the caregivers' need scale. These were considered to be existing needs according to the criteria identified by Longman et al. [17]. Existing needs covered all possible areas on the caregivers' need scale, including personal care, activity management, involvement with health care, work, interpersonal interaction and finance.

\section{Participants' very important needs}

A total of 46 of the 68 existing needs identified (67.7\%) were considered to be "very important". Again, very important needs covered all possible areas on the scale and the highest-rated very important needs were: to be told about symptoms that would be expected as the patient's health changes $(97.6 \%$ of participants); need for a home care or public health nurse (95.0\%); need to be kept informed of patient's condition (94.7\%); help with patient's emotional needs $(87.8 \%)$; and help with additional expenses due to patient care (85.4\%). Table 2 presents the very important needs identified by need area.

\section{Participants' unmet needs}

Atotal of 52 out of 68 (76.4\%) identified needs were marked as "unmet". As with existing and very important needs, the unmet needs also covered all possible areas on the caregivers' need scale. Table 2 presents the unmet needs reported by participants on the caregivers' need scale by need areas. The highest rated unmet needs according to need areas were:

- for personal care "be assured patient is comfortable", "need help knowing how to care for patient" and "be told about symptoms to be expected as patient health changes";

- for activity management: "help assessing his/her ability to give his/her own care (e.g. taking medication or changing a dressing), "help with ways

\begin{tabular}{|c|c|c|}
\hline Characteristic & No. & $\%$ \\
\hline \multicolumn{3}{|l|}{ Sex } \\
\hline Male & 18 & 22.0 \\
\hline Female & 64 & 78.1 \\
\hline \multicolumn{3}{|l|}{ Marital status } \\
\hline Married & 60 & 73.2 \\
\hline Single & 14 & 17.1 \\
\hline Widow & 5 & 6.1 \\
\hline \multirow[t]{2}{*}{ Divorced } & 3 & 3.7 \\
\hline & Mean (SD) & Range \\
\hline Age (years) & $36.2(15.8)$ & $21-62$ \\
\hline Education (years) & $8.4(12.6)$ & $2-18$ \\
\hline Travel distance to hospital (miles) & $32.5(14.6)$ & $20-60$ \\
\hline Time to travel to hospital (min) & $64.0(17.4)$ & $35-120$ \\
\hline Income (US\$) & $380.8(150.6)$ & $100-1800$ \\
\hline No. of people in household & $6.6(4.5)$ & $0-12$ \\
\hline
\end{tabular}

\section{$S D=$ standard deviation}




\begin{tabular}{|c|c|c|c|c|c|c|c|}
\hline \multirow[t]{2}{*}{ Need area } & \multirow{2}{*}{$\begin{array}{c}\text { Total items } \\
\text { No. }\end{array}$} & \multicolumn{2}{|c|}{ Existing needs ${ }^{\mathrm{a}}$} & \multicolumn{2}{|c|}{ Very important needs ${ }^{\mathbf{b}}$} & \multicolumn{2}{|c|}{ Unmet existing needs } \\
\hline & & No. & $\%^{\mathrm{c}}$ & No. & $\%^{\mathrm{d}}$ & No. & $\%^{\mathrm{d}}$ \\
\hline Personal care & 28 & 22 & 78.6 & 13 & 59.1 & 18 & 81.8 \\
\hline Activity management & 13 & 11 & 84.6 & 8 & 72.7 & 11 & 100.0 \\
\hline Involvement with health care & 19 & 13 & 68.4 & 11 & 84.6 & 9 & 69.2 \\
\hline Work & 2 & 2 & 100.0 & 1 & 50.0 & 2 & 100.0 \\
\hline Interpersonal interaction & 21 & 15 & 71.4 & 11 & 73.3 & 10 & 66.7 \\
\hline Finance & 7 & 5 & 71.4 & 2 & 40.0 & 2 & 40.0 \\
\hline
\end{tabular}

${ }^{a}$ Existing needs were items identified by more than $66 \%$ of participants as needs.

${ }^{b}$ Very important needs were those rated by more than $50 \%$ of participants as 9 or 10 on a scale of $0-10$, with 10 the highest. cPercentage of total.

${ }^{d}$ Percentage of existing needs.

of relieving the patient's physical distress", "need information about community resources to help with his/ her activities of daily living (meals, housekeeping, etc.);

- for involvement with health care: "need to be informed of any changes", "need to be kept informed of patient's condition", "need to be closer to the medical centre";

- for work: "need to balance homemaking with patient's needs";

- for interpersonal interaction: "need to share feelings and experiences with others in similar situations", "need for acceptance, support, and comfort from staff", "need to express my own emotions"; and

- for financial: "need to obtain financial assistance for patient".

Table 3 shows the items on the caregivers' need scale identified as very important needs by need area; the highest rate very important needs are marked.

\section{Discussion}

Jordanian caregivers of patients with cancer in this study reported almost double the number of existing needs identified by caregivers in 2 studies in the United States of America (USA) $[17,18]$. Table 4 compares the results of Longman et al. [17] and Silveira and Winstead-Fry [18] with the current results. Very important needs of the urban and rural samples in the USA were identified in only 3 areas (personal care, involvement with health care and interpersonal interaction), while Jordanian caregivers reported very important needs in all 6 areas of the caregivers' need scale (personal care, activity management, involvement with health care, work, interpersonal interaction and finance). About $76.5 \%$ of the existing needs identified were found to be unmet among Jordanian caregivers, as compared with $30 \%$ and $14 \%$ for the urban and rural USA samples respectively. These results suggest that there is insufficient attention given to the needs of caregivers of patients with cancer in Jordan.

The differences in the results can be attributed to several factors. First, there were demographic differences between the 3 samples. For example, the Jordanian participants were younger, less educated, travelled longer and spent more time travelling to hospital than the USA urban and rural samples.

Secondly, there are unique features of the Jordanian health care system. These features include problems with access to care (physical distribution and insurance), inadequate staff and supplies and poor availability of supportive services. Primary and secondary health services are available and accessible to mostJordanians [19]. However, tertiary and specialized services are centralized in major cities, oncology services are limited to 2 major cites, and radiotherapy is available only in the capital, Amman. On average, participants in this study reported that they travelled 60 miles and spend 90 minutes to obtain their required services. Such a problem places extra burdens of cost and effort on patients and their caregivers, which limits their ability to seek help when they need it.

Although about 32\% of Jordanians have no health insurance [19], uninsured oncology patients can obtain medical treatment in public institutions free of charge. Therefore, health insurance appears not to be a problem for such patients. Although Jordanian hospitals employ the highest available technologies and very competent physicians and nurses, there are staff shortages, high number of patients being treated in outpatient chemotherapy clinics and brief contact times between health professionals and patients. These factors limit the supportive and educational services provided to the caregivers of patients.

Researchers reported that Jordanian hospitals are experiencing falling values of salaries, shortages of drugs and supplies, shortages of physicians and nurses and high bed occupancy rates (80\%) [20]. The majority of Jordanian hospitals do not employ support services such as psychologists and social workers. Researchers assert that Arabs are not familiar with the profession of 


\section{Table 3 Items identified as very important needs ${ }^{\mathrm{a}}$ by caregivers of cancer patients}

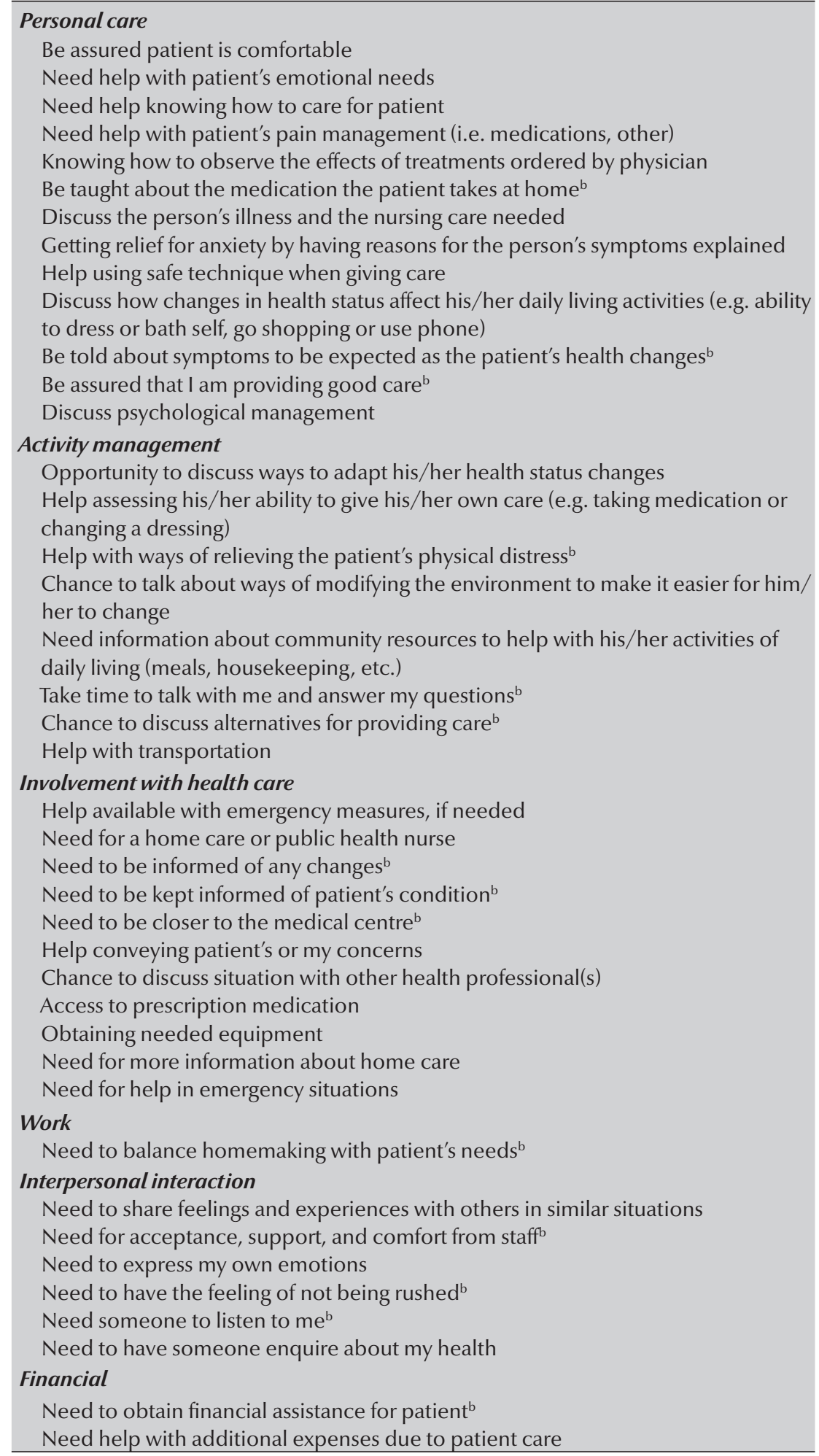

${ }^{a}$ Very important needs were those rated by more than $50 \%$ of participants as 9 or 10 on a scale 0-10, with 10 being the highest.

${ }^{b}$ Highest rated very important needs.

social work, and rely on family members and friends for help and support [21]. In addition, Arabs perceive mental and emotional illnesses as stigmatizing, which limits the use of psychologists in Jordanian hospitals $[21,22]$.

Furthermore, home nursing services, help groups and agencies, and educational materials are very scarce in Jordan, resulting in limited resources when Jordanian caregivers need help and support. Moreover, the fact that caregivers' needs are not incorporated in the nursing care plans further complicates the problem, resulting in caregivers' needs being unexplored and unmanaged.

Finally, cultural issues of Jordanian Arabs may explain the increased number of existing and unmet needs reported by participants in this study. These issues are the high sense of obligation and commitment to the ill, the gaps in communication patterns and the utilization of ineffective traditional treatments. Jordanians, as Muslims, feel they should be very committed to the care of the sick and weak; care for the elderly and sick is regarded as an expression of worship [21,23]. This sense of commitment is expressed at 3 levels: intentions (promises and pledges a person retains deep inside about a specific situation), verbal expressions (words a person uses about the situation) and actions (measures the person takes to implement the intentions) [23]. Consequently, Muslims attempt to reach a state of perfection in their care for loved ones who are sick, which may exacerbate the sense of burden, especially when the care is complicated, such as in the care of cancer patients.

As for communication patterns, caregivers of Jordanian patients with cancer may not report their distress to health care professionals for 3 reasons. First, Jordanian caregivers may not express their sense of distress and burden to avoid being perceived by the patients as if they are trying to quit. Secondly, Muslims are encouraged to meet hardships, illness and death with patience and prayer, and to avoid excessive complaints. Thirdly, Arabs regard revealing family issues to strangers as a sign of mistrust and weakness [22]. Fourthly, when a treatment plan is not suited to an Arab client, it will result in noncompliance with the instructions 


\begin{tabular}{lcccccc}
\hline Table 4 Needs identified as very important ${ }^{\text {a }}$ by caregivers of cancer patients in different studies & & \\
Need area & Urban area & USA & (1992) [18] & Rural area USA (1997) [19] & Current study Jordan (2006) \\
& No. & $\%$ & No. & $\%$ & No. & $\%$ \\
Personal care & 4 & 22 & 5 & 18 & 13 & 59 \\
Activity management & 0 & 0 & 1 & 8 & 8 & 73 \\
Involvement with health care & 2 & 17 & 3 & 16 & 11 & 85 \\
Work & 0 & 0 & 0 & 0 & 1 & 50 \\
Interpersonal interaction & 4 & 19 & 4 & 19 & 11 & 87 \\
Finance & 0 & 0 & 0 & 0 & 2 & 40 \\
\hline
\end{tabular}

${ }^{a}$ Very important needs were those rated by more than $50 \%$ of participants as 9 or 10 on a scale of 0-10, with 10 being the highest. USA = United States of America.

instead of negotiation [24]. Other authors assert that Arab patients seek medications from pharmacists without prescriptions and become disappointed if they are not treated with a variety of medications for the same illness [21]. Finally, Arab clients prefer more aggressive treatments over teaching and counselling. For example, they prefer injections to tablets, and intravenous to intramuscular treatments [24].
There were some limitations to the current study. It utilized a small nonrepresentative sample, which limits the generalizability of the results. Although the caregivers' need scale has been demonstrated to be applicable to other cultures, it may lack cultural sensitivity to capture the unique features of the Jordan Arab culture.

Inspiteofthelimitations ofthisstudy we can recommend that caregivers in
Jordan would benefit from measures to satisfy their unmet needs, including the establishment of a home health care nursing system; facilitation of travel from their home to hospitals; written teaching materials to help patients and caregivers understand the disease process, treatment options and self-care strategies; and the establishment of formal and informal community support systems.

\section{References}

1. Glajchen M. The emerging role and needs of family caregivers in cancer care. Journal of Supportive Oncology, 2004, 2(2):145-155.

2. Sherwood P et al. Caregivers of persons with a brain tumor: a conceptual model. Nursing Inquiry, 2004, 11(1):43-53.

3. Wilkinson AM, Lynn J. Caregiving for advanced chronic illness patients. Techniques in Regional Anesthesia and Pain Management, 2005, 9(3):122-132.

4. Nijboer $\mathrm{C}$ et al. Cancer and caregiving: the impact on the caregiver's health, Psycho-Oncology, 1998, 7:3-13.

5. Sharpe L et al. The relationship between available support, unmet needs and caregiver burden in patients with advanced cancer and their carers. Psycho-Oncology, 2005, 14:102 -114.

6. Giarelli E, McCorkle R, Monturo C. Caring for a spouse after prostate surgery: the preparedness needs of wives. Journal of Family Nursing, 2003, 9(4):453-485.

7. Schumacher KL et al. Family caregiving skill: development of the concept. Research in Nursing and Health, 2000, 23:191203.

8. Schulz R, Beach SR. Caregiving as a risk factor for mortality: the Caregiver Health Effects Study. Journal of the American Medical Association, 1999, 282:2215-2219.

9. Clipp E, Moore M. Caregiver time use: an outcome measure in clinical trial research on Alzheimer's disease. Clinical Pharmacology and Therapeutics, 1995, 58:228-236.

10. Jensen S, Given BA. Fatigue affecting family care-givers of cancer patients. Cancer Nursing, 1991, 14:181-187.

11. Stetz KM. Caregiving demands during advanced cancer: the spouse's needs. Cancer Nursing, 1987, 10:260-268.
12. Weitzner MA, McMillan SC, Jacobsen PB. Family caregiver quality of life: differences between curative and palliative cancer treatment settings. Journal of Pain and Symptom Management, 1999, 17(6):418-427.

13. Miaskowski $\mathrm{C}$ et al. Differences in mood states, health status and caregiver strain between family caregivers of oncology patients with and without cancer-related pain. Journal of Pain and Symptom Management, 1997, 13:138-147.

14. Siegel $\mathrm{K}$ et al. Caregiver burden and unmet patient needs. Cancer, 1991, 68:1131-1140.

15. Kurtz MJ et al. Relationship of caregiver reactions and depression to cancer patient's symptoms, functional states and depression: a longitudinal view. Social Science and Medicine, 1995, 40:837-846.

16. Children's Leukemia Foundation of Michigan. Exploring the needs of cancer patients and caregivers: an overview of key needs assessment findings in 2004 (http://www.leukemiamichigan. org. accessed 21 June 2010).

17. Longman AJ et al. Care needs of home-based cancer patients and their caregivers: quantitative findings. Cancer Nursing, 1992, 15(3):182-190.

18. Silveira JM, Winstead-Fry P. The needs of patients with cancer and their caregivers in rural areas. Oncology Nursing Forum, 1997, 24(1):71-76.

19. Bateiha A. Situation analysis of the health sector in Jordan. (http://www.hhc.gov.jo/sa.htm, accessed 14 July 2010).

20. Franco LM et al. Health worker motivation in Jordan and Georgia: a synthesis of results. Applied research 5, Technical paper 3. Bethesda, Maryland, Partnerships for Health Reform Project, Abt Associates Inc. 2000. 
21. Ahmad NM. Arab-American culture and health care. (http:// www.case.edu/med/epidbio/mphp439/Arab-Americans. htm, accessed 21 June 2010).

22. Al-Krenawi A, Graham, JR. Culturally sensitive social work practice with Arab clients in mental health settings. Health and Social Work, 2006, 25(1):9-22.

23. Rasssool GH. The crescent and Islam: healing, nursing, and spiritual dimension. Some consideration toward understand- ing of the Islamic perspectives on caring. Journal of Advanced Nursing, 2000, 32(6):1476-1484.

24. Hammad A et al. ACCESS guide to Arab culture: health care delivery to the Arab American Community. Dearborn, Michigan, Arab Community Center for Economic and Social Services, 1999.

\section{Women's health}

A number of advances have been made over the past few years in respect to women's health issues, and in fact, such issues are more openly discussed nowadays and women themselves are more involved in controlling their own health. Although there is still no room for complacency, there has been significant progress in opportunities for health education and treatment, including preventive treatment.

In her inaugural speech to the World Health Assembly in 2006, Margaret Chan, Director General of the World Health Organization, spoke at length on this topic:

"The health of women must be the other key indicator - and I do not mean just maternal health. Women do much more than have babies. Unfortunately, their activities in households and communities, coupled with their low status, make them especially vulnerable to health problems - from indoor air pollution and multiple infectious diseases to violence.

Yet evidence from many sources also shows that women are agents of change - for families, the workforce, and entire communities. The health of children largely depends on the health of women. As mothers, as sisters, as aunts and as grandmothers in the home. As carers, as teachers and as health workers in the community. And I have been all of these - except a grandmother!

Reducing health problems in women and empowering them will result in a dramatic increase in health-promoting behaviours - right where it counts most."

(Source: http://www.who.int/dg/speeches/2006/wha/en/index.html) 